\title{
Giant Exciton Mott Density in Anatase $\mathrm{TiO}_{2}$
}

\author{
Edoardo Baldini ${ }^{1,2, *}$ Tania Palmieri® ${ }^{1}$ Adriel Dominguez, ${ }^{3,4,5}$ Angel Rubio, ${ }^{6,7,8}$ and Majed Chergui® ${ }^{1, \dagger}$ \\ ${ }^{1}$ Laboratory of Ultrafast Spectroscopy, ISIC and Lausanne Centre for Ultrafast Science (LACUS), \\ École Polytechnique Fédérale de Lausanne, CH-1015 Lausanne, Switzerland \\ ${ }^{2}$ Department of Physics, Massachusetts Institute of Technology, 02139 Cambridge, Massachusetts, USA \\ ${ }^{3}$ Bremen Center for Computational Material Science (BCCMS), Bremen 28359, Germany \\ ${ }^{4}$ Shenzhen JL Computational Science and Applied Research Institute (CSAR), Shenzhen 518110, China \\ ${ }^{5}$ Beijing Computational Research Center (CSRC), Beijing 100193, China \\ ${ }^{6}$ Max Planck Institute for the Structure and Dynamics of Matter, Hamburg 22761, Germany \\ ${ }^{7}$ Departamento Física de Materiales, Universidad del País Vasco, Avenida Tolosa 72, E-20018 San Sebastian, Spain \\ ${ }^{8}$ Center for Computational Quantum Physics, The Flatiron Institute, 162 Fifth Avenue, New York, New York 10010, USA
}

(Received 27 February 2020; revised 4 July 2020; accepted 17 August 2020; published 10 September 2020)

\begin{abstract}
Elucidating the carrier density at which strongly bound excitons dissociate into a plasma of uncorrelated electron-hole pairs is a central topic in the many-body physics of semiconductors. However, there is a lack of information on the high-density response of excitons absorbing in the near-to-mid ultraviolet, due to the absence of suitable experimental probes in this elusive spectral range. Here, we present a unique combination of many-body perturbation theory and state-of-the-art ultrafast broadband ultraviolet spectroscopy to unveil the interplay between the ultraviolet-absorbing two-dimensional excitons of anatase $\mathrm{TiO}_{2}$ and a sea of electron-hole pairs. We discover that the critical density for the exciton Mott transition in this material is the highest ever reported in semiconductors. These results deepen our knowledge of the exciton Mott transition and pave the route toward the investigation of the exciton phase diagram in a variety of wide-gap insulators.
\end{abstract}

DOI: 10.1103/PhysRevLett.125.116403

One of the major intellectual advancements in modern condensed matter physics has been the formulation of the insulator-to-metal transition, first given by Mott [1]. In the simplest picture, increasing the carrier density in an insulator above a critical value-known as the Mott density $\left(n_{M}\right)$-leads to the transformation of bound states into delocalized states, eventually turning the material into a conductor. The consequences of this theoretical prediction have been far-reaching, revealing unprecedented insights into the properties of solids such as chemically doped and photodoped band semiconductors [2,3], excitonic insulators [4], and strongly correlated electron systems [5].

In the case of a photodoped band semiconductor, the bound states are represented by excitons, collective excitations of electron-hole $(\mathrm{e}-\mathrm{h})$ pairs coupled via the longrange Coulomb interaction. Increasing the carrier density reinforces the fermionic coupling among the electrons and holes, ultimately resulting in the dissociation of the bound states above $n_{M}$. The Mott criterion here predicts that the transition occurs if $k_{S} \cdot a_{B} \simeq 1.19$, where $k_{S}$ is the critical

Published by the American Physical Society under the terms of the Creative Commons Attribution 4.0 International license. Further distribution of this work must maintain attribution to the author(s) and the published article's title, journal citation, and DOI. screening length at which bound states nominally disappear and $a_{B}$ is the exciton Bohr radius [2]. More refined theoretical analysis $[4,6]$ and extensive experimental work [7-13] have instead revealed a rich phase diagram of exotic states persisting above $n_{M}$. Notable examples include robust excitonic and biexcitonic correlations [7-9], emergent Mahan excitons [10], anomalous metallic states [11], e-h droplets [12,13], and possible Bose condensates of photoexcited e-h Cooper pairs [4]. Therefore, the identification of $n_{M}$ in semiconductors acquires a crucial importance for discovering hitherto unobserved phenonena and clarifying how excitons react to the large carrier densities present in many optoelectronic devices.

One solid that has recently emerged as a promising platform to explore bound exciton physics is the anatase polymorph of $\mathrm{TiO}_{2}$ [14-16], a material extensively used in light-energy conversion applications [17,18] and transparent conducting substrates [19]. This system is an indirect gap insulator [Fig. 1(a)]: the valence band (VB) top is close to the $X$ point of the Brillouin zone, whereas the conduction band (CB) bottom lies at the $\Gamma$ point. The optical spectrum is dominated by a prominent direct excitation around $3.80 \mathrm{eV}$ [peak I in Fig. 1(b)], which lies on the tail of indirect interband transitions (similar to bulk transition metal dichalcogenides [20]). Since the energy of peak I is significantly lower than the direct quasiparticle 
(a)

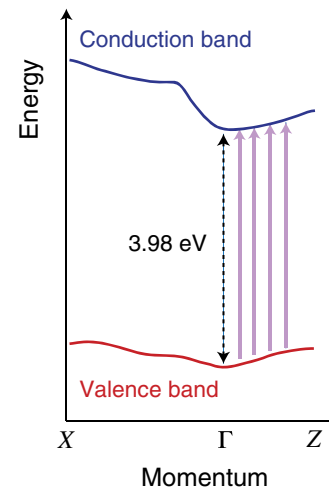

(b)

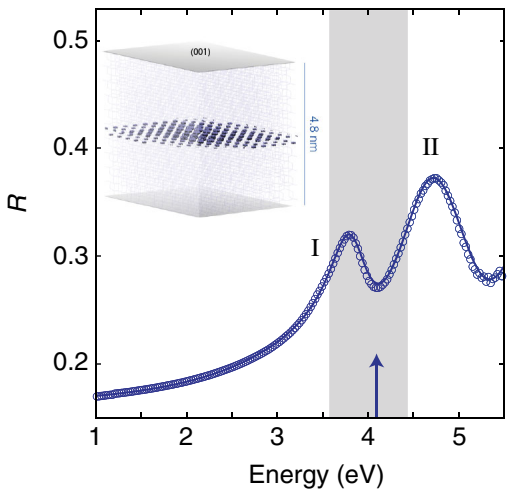

FIG. 1. (a) Schematic illustration of the electronic band structure of anatase $\mathrm{TiO}_{2}$, as obtained from $G W$ calculations. The direct gap around the $\Gamma$ point is determined in experiments to be $\sim 3.98 \mathrm{eV} \mathrm{[14].} \mathrm{The} \mathrm{violet} \mathrm{arrows} \mathrm{indicate} \mathrm{the} \mathrm{single-particle}$ states that contribute to building up the $a$-axis bound exciton transition. (b) Reflectivity spectrum of anatase $\mathrm{TiO}_{2}$ at RT with the light electric field polarized along the $a$ axis. Peak I is the 2D bound exciton, whereas peak II is a high-energy resonant exciton. The data, measured by spectroscopic ellipsometry, and their assignment are obtained from Ref. [14]. The pump photon energy of $4.10 \mathrm{eV}$ used for the pump-probe experiment is indicated by the blue arrow and the probed region is highlighted as a grey shaded area. The inset shows the wave function of the bound 2D exciton around $3.79 \mathrm{eV}$. The isosurface representation shows the electronic configuration when the hole of the considered excitonic pair is localized close to one oxygen atom. The colored region represents the excitonic squared modulus wave function.

gap of $3.98 \mathrm{eV}$, this transition is a rare type of strongly bound exciton with binding energy $\left(E_{B}\right)$ larger than $150 \mathrm{meV}$ [14]. Such a large $E_{B}$ stems from the contribution of many single-particle states in building up the exciton wave function along the $\Gamma-Z$ symmetry direction, where the VB and CB have almost parallel dispersion [violet arrows in Fig. 1(a)]. Calculations reveal that these excitons have an intermediate character between the Wannier and the Frenkel limit, and are characterized by a two-dimensional (2D) wave function in the three-dimensional (3D) lattice [inset to Fig. 1(b)] [14]. The large $E_{B}$ makes them particularly immune to perturbations, such as temperature or the scattering at impurities and defects. As a result, these collective excitations manifest themselves also in the room temperature (RT) absorption spectrum of the defect-rich nanoparticles used in typical light-conversion applications $[14,15,21]$. However, the extent to which these excitons persist against a high density of free carriers injected in the bands is yet to be addressed. On a fundamental side, shedding light on this problem would show how different many-body effects conspire to destabilize a 2D bound state in a 3D crystal and establish whether the exciton can form stable polaritonic states in ad-hoc-designed microcavities. On the technological side, a deeper knowledge of $n_{M}$

would guide the rational design of effective transparent conducting substrates based on $\mathrm{TiO}_{2}$.

Nevertheless, addressing this problem poses considerable challenges to currently available theoretical and experimental techniques. Theoretically, one should build realistic models of the material's electronic and optical properties that account for the plethora of many-body processes induced by the free carriers. In this respect, many-body perturbation theory has revolutionalized the description of the equilibrium electrodynamical properties of materials [22], but its application to doped semiconductors is still in its infancy [23,24]. Experimentally, one would need an accurate method to inject a high density of free carriers and monitor the modification of the exciton optical line shape. This task cannot be accomplished by measuring the optical response of the material upon chemical doping, since the description of the Mott transition becomes more intricate [3], with the dopant-induced inhomogeneous broadening $[25,26]$ and possible electronelectron correlations [27] masking the effects induced by the free-carrier density. A more accurate approach relies on photodoping the crystal out of equilibrium using an abovegap laser pulse and mapping the optical response around the exciton resonance with subpicosecond time resolution. Unlike its steady-state analog, this technique allows for disentangling the contributions of different optical nonlinearities on the exciton peak, based on their characteristic timescale. In $\mathrm{TiO}_{2}$, this would require the simultaneous generation of intense pump and broadband probe pulses covering the elusive near-to-mid-ultravoilet (UV) range $(3.20-4.50 \mathrm{eV})$, a technology that has long been limited by constraints in nonlinear optical conversion schemes [28-31].

In this Letter, we set a first milestone toward the determination of $n_{M}$ for a bound exciton absorbing UV light, in anatase $\mathrm{TiO}_{2}$ single crystals, via a unique combination of many-body perturbation theory and state-of-theart ultrafast broadband UV spectroscopy. We reveal that the $2 \mathrm{D}$ excitons are stable bound quasiparticles in the material at least up to a giant carrier density of $\sim 5 \times 10^{19} \mathrm{~cm}^{-3}$ at RT. Our results show that the bound states in $\mathrm{TiO}_{2}$ are among the most robust excitons ever reported, opening intriguing perspectives for the study of many-body e-h correlations in a wide class of hitherto inaccessible insulators.

As a first step in our study, we explore theoretically the interplay between the bound 2D excitons of anatase $\mathrm{TiO}_{2}$ and free carriers by computing the $G W$ band structure within the frozen lattice approximation in a uniformly electron-doped crystal. Thereafter, we obtain the optical response in the presence of e-h correlations by solving the Bethe-Salpeter equation at the different doping levels [22,32]. More details about the method and the validity of the approximations used are provided in the Supplementary Material [33]. A thorough comparison 
between the theoretical and experimental response at zero doping was given in Ref. [14]. In Fig. 2(a) we only focus on the doping $(n)$ dependence, which shows a strongly nonlinear response of the single-particle gap (dashed vertical lines) and the optical spectra with $n$. In particular, we find that the quasiparticle gap and exciton absorption of the system do not change between $n=0 \mathrm{~cm}^{-3}$ and $n \sim 1.4 \times 10^{19} \mathrm{~cm}^{-3}$. At $n=14 \times 10^{19} \mathrm{~cm}^{-3}$, the exciton peak blueshifts by $\sim 50 \mathrm{meV}$, a value that is larger than the carrier-induced blueshift of the quasiparticle gap $(\sim 20 \mathrm{meV})$. As a result, $E_{B}$ is weakened by $\sim 30 \mathrm{meV}$. Increasing $n$ to $35 \times 10^{19} \mathrm{~cm}^{-3}$ results in an abrupt and large redshift of the quasiparticle gap due to band gap renormalization (BGR). Here, the quasiparticle gap overlaps the exciton peak energy, signaling the occurrence of the Mott transition. However, even if bound states cease to exist above $n_{M}$, excitonic correlations still persist in the form of a resonant exciton that shapes the optical response. Further increasing $n$ results in a substantial smearing of this resonant exciton and in the shrinking of the quasiparticle gap. The complete dependence of $E_{B}$ on $n$ is shown in Fig. 2(b). From this plot, we estimate that the Mott transition occurs at a surprisingly high value of $n_{M} \sim 35 \times 10^{19} \mathrm{~cm}^{-3}$.

Next, we investigate this finding experimentally using ultrafast spectroscopy with a near-to-mid-UV continuum probe. The description of the experimental methods is (a)

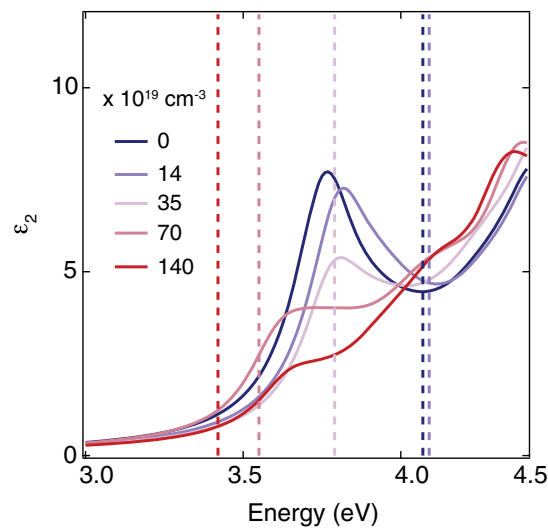

(b)

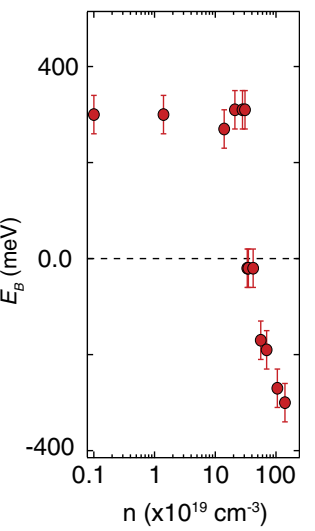

FIG. 2. (a) Imaginary part of the $a$-axis dielectric function of anatase $\mathrm{TiO}_{2}$, calculated by solving the Bethe-Salpeter equation for pristine and $n$-doped $\mathrm{TiO}_{2}$. The optical response of the $n$ doped crystal with $n=1.4 \times 10^{19} \mathrm{~cm}^{-3}$ (not shown) overlaps almost completely that of the pristine case, indicating that this doping level does not affect the exciton peak energy. Only when $n>10 \times 10^{19} \mathrm{~cm}^{-3}$, does the exciton blueshift. The vertical lines represent the quasiparticle gap energy at each doping level. The Mott transition occurs at $n_{M} \sim 35 \times 10^{19} \mathrm{~cm}^{-3}$; i.e., it occurs when the quasiparticle gap matches the exciton peak energy. (b) Doping dependence of $E_{B}$, estimated from the energy difference between the quasiparticle gap energy and the exciton peak energy. An abrupt change is observed around $n>10 \times$ $10^{19} \mathrm{~cm}^{-3}$ and the bound states are lost at $n_{M}$. given in the Supplemental Material [33]. Our goal is to map the response of the bound exciton in $\mathrm{TiO}_{2}$ upon illumination with an intense laser pulse centered around $4.10 \mathrm{eV}$ [blue arrow in Fig. 1(b)]. This photon energy lies above the exciton peak and thus excites uncorrelated e-h pairs in the solid. We set the incident fluence to the maximum value that our state-of-the-art apparatus can deliver and we carefully convert it into a density of photoexcited carriers (see the Supplemental Material [33] for the estimate of the uncertainties). We obtain $n \sim 5 \times 10^{19} \mathrm{~cm}^{-3}$, i.e. below the theoretically predicted $n_{M}$ but sufficiently high compared to the density at which excitons dissociate in most solids [10,62-65]. Subsequently, we monitor the relative changes in the material reflectivity $(\Delta R / R)$ over a broad spectral range covering the bound exciton feature [grey shaded area in Fig. 1(b)]. Depending on the spectral extension of our probe pulse, the time resolution of the setup varies between 80 fs and 1 ps [29].

Figure 3(a) displays the color-coded map of $\Delta R / R$ as a function of the probe photon energy and pump-probe time delay. To allow for a broadband detection between 3.60 and $4.40 \mathrm{eV}$, the time resolution of the setup is set at $700 \mathrm{fs}$. We observe that the signal is positive above $\sim 3.95 \mathrm{eV}$ and negative below this energy. The zero-crossing point varies with time, suggesting a change in the peak position and linewidth of the exciton peak. To visualize these changes, we reconstruct the pump-induced temporal evolution of the material's reflectivity by combining our steady-state and time-resolved optical data. The results, shown in Fig. 3(b), indicate that upon photoexcitation the exciton band decreases its absolute reflectivity, its linewidth broadens, and it shifts to the blue. The wide spectral region covered by this measurement enables us to perform a quantitative analysis of the reflectivity data and obtain the corresponding absorption spectra at different time delays. To this aim, we fit the steady-state optical data with a Lorentz model, as shown by the solid line in Fig. 1(b). Thereafter, we describe the pump-induced changes of the reflectivity spectrum through the variation of the bound exciton parameters (details are given in the Supplemental Material [33]). Iterating the fit at each time delay yields the time-dependent absorption coefficient $\alpha(\omega, \mathrm{t})$ [Fig. 3(c)], as well as the time evolution of the exciton oscillator strength, linewidth, and peak energy [Figs. 3(d)-3(f)]. At the present photoexcited carrier density, we find that the exciton oscillator strength decreases by only $\sim 5 \%$ [Fig. 3(d)] within our time resolution and recovers with a biexponential trend with timescales of $0.85 \pm 0.37$ ps and $33 \pm 7$ ps. In contrast, a different temporal behavior is shared by the exciton peak energy [Fig. 3(e)], and linewidth [Fig. 3(f)]. In particular, the exciton peak energy increases by $\sim 35 \mathrm{meV}$ and recovers on timescales of $20 \pm 4$ ps and $250 \pm 111$ ps. This suggests that the same optical nonlinearity causes both the linewidth and peak energy increase. 
(a)

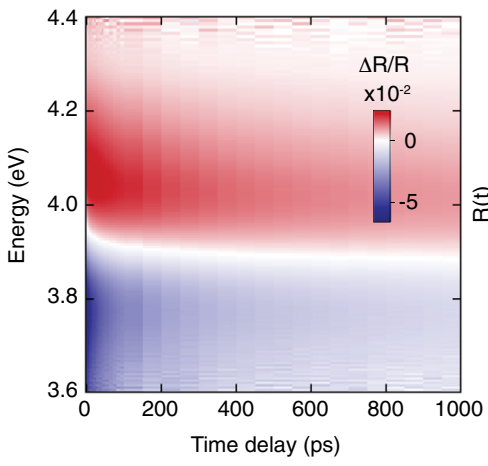

(d)

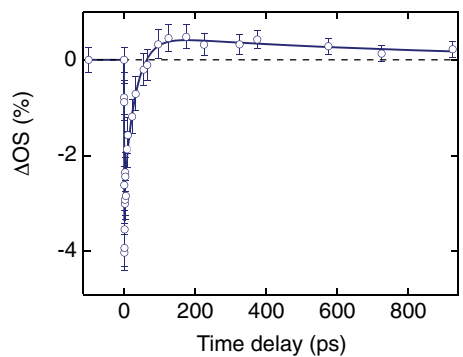

(b)

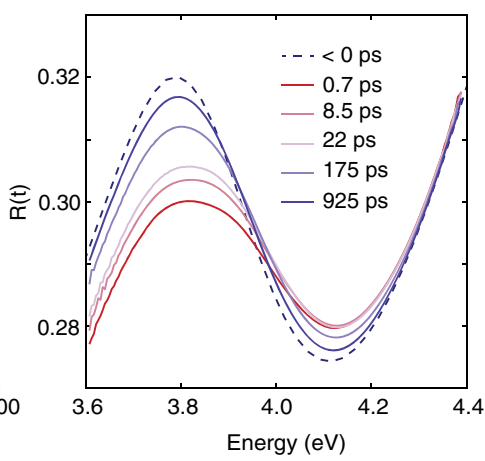

(e)

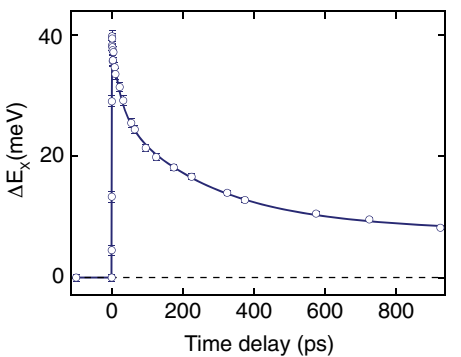

(c)

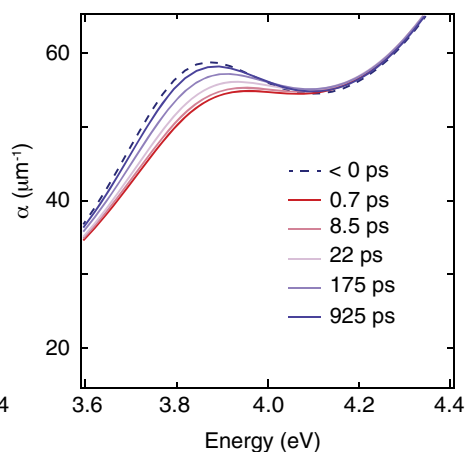

(f)

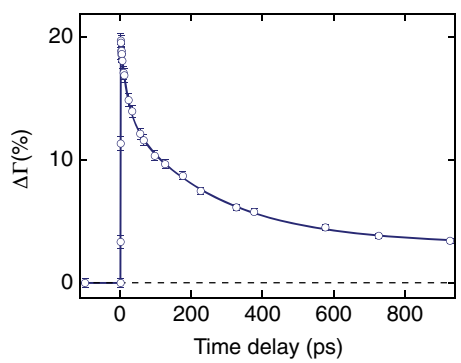

FIG. 3. (a) Color-coded map of $\Delta R / R(\omega, \mathrm{t})$ as a function of probe photon energy and time delay between pump and probe. (b) Spectral evolution of $R(\omega, \mathrm{t})$, obtained by combining the steady-state $R(\omega)$ and the $\Delta R / R(\omega, \mathrm{t})$ data in the time window $700 \mathrm{fs}-1 \mathrm{~ns}$. The $\mathrm{R}$ of the unperturbed system is displayed as a dashed blue line. (c) Spectral evolution of $\alpha(\omega, \mathrm{t})$, obtained as a result of the Lorentz fit in the time window $700 \mathrm{fs}-1 \mathrm{~ns}$. The $\alpha$ of the unperturbed system is displayed as a dashed blue line. (d)-(f) Temporal evolution of (c) the oscillator strength, (d) the linewidth, and (e) the peak energy of the bound exciton upon photoexcitation.

The spectrotemporal analysis of the exciton line shape allows us to disentangle the single-particle and many-body effects participating in the exciton optical nonlinearities [66]. In particular, the presence of $e-h$ populations inside the bands partially block the transitions contributing to the exciton state [indicated by the violet arrows in Fig. 1(a)]. As a result, this phase-space filling (PSF) of the relevant single-particle states causes a decrease in the exciton oscillator strength. Owing to the characteristic electronic structure of anatase $\mathrm{TiO}_{2}$ [Fig. 1(a)], the photoexcited electrons relax to the bottom of the $\mathrm{CB}$ at $\Gamma$, whereas the holes to the top of the VB close to $X$. As such, the PSF contribution to the exciton spectral changes arises exclusively from an electron population close to the bottom of the $\mathrm{CB}$. Moreover, the increased broadening of the exciton linewidth is a manifestation of long-range Coulomb screening (CS), as the photoexcited carrier density reduces the exciton lifetime [21]. Finally, the origin of the exciton blueshift over time deserves deeper attention. Three known optical nonlinearities can cause a shift in an exciton peak upon above-gap illumination, namely BGR, CS, and PSF $[66,67]$. BGR leads to a density-dependent shrinkage of the single-particle states and hence a redshift of the exciton feature due to the change in electron-electron and electronion interaction. Long-range CS modifies the e-h interaction, resulting in a reduced $E_{B}$ and in a blueshift of the exciton peak. Finally, PSF may also contribute to the blueshift of the exciton peak: indeed, the carriers populating the band edges can lead to the apparent shift of the overall absorption edge toward high energies (Burstein-Moss effect) [68,69]. These processes act simultaneously on the exciton peak energy, their relative weights being governed by the material parameters and dimensionality [66,67]. However, under the present excitation conditions, the exciton peak energy temporal response closely resembles that of its linewidth. This strongly indicates that long-range $\mathrm{CS}$ is the dominant nonlinearity behind the exciton blueshift, ruling out PSF and BGR. Therefore, the detected exciton blueshift can be directly correlated with the absolute change in $E_{B}$ produced by the photoexcited e-h plasma. Since $E_{B}$ changes only by $\sim 35 \mathrm{meV}$ at $700 \mathrm{fs}$, excitons are bound entities at this time delay.

As in $\mathrm{TiO}_{2}$ the intraband carrier relaxation is complete within $50 \mathrm{fs}$ (due to the strong electron-phonon coupling) [70,71], one may argue that the results obtained at $700 \mathrm{fs}$ are not representative of the stated excitation density. Indeed, recombination mechanisms such as carrier trapping or three-body Auger processes may have already decreased the actual carrier density contributing to the exciton screening in this indirect-gap material. This requires one to resolve a well-defined exciton feature at a time delay close to $50 \mathrm{fs}$, demonstrating the persistence of the e-h correlations at such a short timescale. Due to the trade-off between time resolution and probe spectral coverage in our 
setup, we also demonstrate the stability of the excitonic correlations at 120 fs by resolving their signature over a narrower range in the reflectivity spectrum. The results, reported in Fig. S5 [33], indicate that the exciton peak is not entirely suppressed by the presence of the e-h plasma and that the excitonic correlations are still intact in this highly non-equilibrium phase.

We believe that the photoexcited carrier density in our experiment lies below the actual value of $n_{M}$. Persistence of excitonic correlations above $n_{M}$ have been recently demonstrated in several semiconductors $[7,8,10]$ and the emergence of Mahan excitons has been invoked [10]. However, in such a scenario, the Wannier exciton feature would be accompanied by the enhancement of the continuum absorption, which is instead absent in our data. Therefore, our result support a scenario in which the actual $n_{M}$ is larger than $5 \times 10^{19} \mathrm{~cm}^{-3}$. Our many-body perturbation theory does not account for finite temperature effects and the presence of quasi-Fermi energies for a nonequilibrium distribution of e-h pairs. Future extensions of our theory to include these effects will refine the theoretically predicted $n_{M}$, most likely around lower values. Despite these corrections, we can confidently conclude that the Mott transition occurs in anatase $\mathrm{TiO}_{2}$ at a remarkably high $n_{M}$. For comparison, other bulk insulators supporting bound excitons built upon the single-particle states have $n_{M}$ varying between $7 \times$ $10^{16} \mathrm{~cm}^{-3}$ and $6.4 \times 10^{18} \mathrm{~cm}^{-3}$ (see Table S1 [33]). In the case of $\mathrm{TiO}_{2}$, such a high $n_{M}$ can be explained by the robustness of the single-particle gap to the injected carrier density, consistent with our calculations and observations by angle-resolved photoemission spectroscopy [14].

In conclusion, our work demonstrates the robustness of the bound excitons in $\mathrm{TiO}_{2}$ and shows the power of ultrafast broadband UV spectroscopy to investigate many-body phenomena involving high-energy excitons and large carrier densities. We envision the application of this method to study a variety of high-energy excitons that strongly couple to the lattice or the spin degrees of freedom, i.e., in perovskite titanates $[72,73]$ or in antiferromagnetic Mott insulators [74].

We thank Alexander Steinhoff and David Snoke for insighful discussions and comments, and Simon Moser and Marco Grioni for providing the sample used for this study. We acknowledge support by the Swiss National Science Foundation via the NCCR:MUST, Contract No. 154056 (PNR 70, "Energy turnaround") and the R'EQUIP Contract No. 206021-157773. We acknowledge financial support from the European Research Council (Grant No. ERC-2015-AdG-694097), the Deutsche Forschungsgemeinschaft (DFG) through RTG2247, and the Cluster of Excellence Advanced Imaging of Matter (AIM) Grant No. EXC 336 2056-390715994. The Flatiron Institute is a division of the Simons Foundation. Support by the Max Planck Institute-New York City Center for Non-Equilibrium Quantum Phenomena is acknowledged.

*ebaldini@mit.edu

†majed.chergui@epfl.ch

[1] N. Mott, Rev. Mod. Phys. 40, 677 (1968).

[2] C. F. Klingshirn, Semiconductor Optics (Springer Science \& Business Media, New York, 2012).

[3] D. Snoke, Solid State Commun. 146, 73 (2008).

[4] S. A. Moskalenko and D. Snoke, Bose-Einstein Condensation of Excitons and Biexcitons: And Coherent Nonlinear Optics with Excitons (Cambridge University Press, Cambridge, England, 2000).

[5] D. Khomskii, Transition Metal Compounds (Cambridge University Press, Cambridge, England, 2014).

[6] D. Guerci, M. Capone, and M. Fabrizio, Phys. Rev. Mater. 3, 054605 (2019).

[7] T. Suzuki and R. Shimano, Phys. Rev. Lett. 109, 046402 (2012)

[8] F. Sekiguchi and R. Shimano, Phys. Rev. B 91, 155202 (2015).

[9] M. Shahmohammadi, G. Jacopin, G. Rossbach, J. Levrat, E. Feltin, J.-F. Carlin, J.-D. Ganiere, R. Butté, N. Grandjean, and B. Deveaud, Nat. Commun. 5, 5251 (2014).

[10] T. Palmieri, E. Baldini, A. Steinhoff, A. Akrap, M. Kollár, E. Horváth, L. Forró, F. Jahnke, and M. Chergui, Nat. Commun. 11, 850 (2020).

[11] F. Sekiguchi, T. Mochizuki, C. Kim, H. Akiyama, L. N. Pfeiffer, K. W. West, and R. Shimano, Phys. Rev. Lett. 118, 067401 (2017).

[12] J. Omachi, T. Suzuki, K. Kato, N. Naka, K. Yoshioka, and M. Kuwata-Gonokami, Phys. Rev. Lett. 111, 026402 (2013).

[13] A. E. Almand-Hunter, H. Li, S. T. Cundiff, M. Mootz, M. Kira, and S. W. Koch, Nature (London) 506, 471 (2014).

[14] E. Baldini, L. Chiodo, A. Dominguez, M. Palummo, S. Moser, M. Yazdi-Rizi, G. Auböck, B. P. P. Mallett, H. Berger, A. Magrez, C. Bernhard, M. Grioni, A. Rubio, and M. Chergui, Nat. Commun. 8, 13 (2017).

[15] E. Baldini, T. Palmieri, A. Dominguez, P. Ruello, A. Rubio, and M. Chergui, Nano Lett. 18, 5007 (2018).

[16] E. Baldini, A. Dominguez, T. Palmieri, O. Cannelli, A. Rubio, P. Ruello, and M. Chergui, Sci. Adv. 5, eaax2937 (2019).

[17] A. Fujishima and K. Honda, Nature (London) 238, 37 (1972).

[18] B. O'Reagen and M. Grätzel, Nature (London) 353, 737 (1991).

[19] Y. Furubayashi, T. Hitosugi, Y. Yamamoto, K. Inaba, G. Kinoda, Y. Hirose, T. Shimada, and T. Hasegawa, Appl. Phys. Lett. 86, 252101 (2005).

[20] A. Molina-Sánchez, D. Sangalli, K. Hummer, A. Marini, and L. Wirtz, Phys. Rev. B 88, 045412 (2013).

[21] E. Baldini, T. Palmieri, T. Rossi, M. Oppermann, E. Pomarico, G. Auböck, and M. Chergui, J. Am. Chem. Soc. 139, 11584 (2017).

[22] G. Onida, L. Reining, and A. Rubio, Rev. Mod. Phys. 74, 601 (2002). 
[23] A. Schleife, C. Rödl, F. Fuchs, K. Hannewald, and F. Bechstedt, Phys. Rev. Lett. 107, 236405 (2011).

[24] E. A. Pogna, M. Marsili, D. De Fazio, S. Dal Conte, C. Manzoni, D. Sangalli, D. Yoon, A. Lombardo, A. C. Ferrari, A. Marini, G. Cerullo, and D. Prezzi, ACS Nano 10, 1182 (2016).

[25] T. Makino, K. Tamura, C. Chia, Y. Segawa, M. Kawasaki, A. Ohtomo, and H. Koinuma, Phys. Rev. B 65, 121201(R) (2002).

[26] M. Feneberg, S. Osterburg, K. Lange, C. Lidig, B. Garke, R. Goldhahn, E. Richter, C. Netzel, M. D. Neumann, N. Esser et al., Phys. Rev. B 90, 075203 (2014).

[27] Z. Yong, P. E. Trevisanutto, L. Chiodo, I. Santoso, A. R. Barman, T. C. Asmara, S. Dhar, A. Kotlov, A. Terentjevs, F. Della Sala, V. Olevano, M. Rubhausen, T. Venkatesan, and A. Rusydi, Phys. Rev. B 93, 205118 (2016).

[28] T. Shih, E. Mazur, J.-P. Richters, J. Gutowski, and T. Voss, J. Appl. Phys. 109, 043504 (2011).

[29] G. Auböck, C. Consani, F. van Mourik, and M. Chergui, Opt. Lett. 37, 2337 (2012).

[30] B. A. West and A. M. Moran, J. Phys. Chem. Lett. 3, 2575 (2012).

[31] R. Borrego-Varillas, A. Nenov, L. Ganzer, A. Oriana, C. Manzoni, A. Tolomelli, I. Rivalta, S. Mukamel, M. Garavelli, and G. Cerullo, Chem. Sci. 10, 9907 (2019).

[32] L. Hedin, Phys. Rev. 139, A796 (1965).

[33] See the Supplemental Material at http://link.aps.org/ supplemental/10.1103/PhysRevLett.125.116403 for additional analysis and discussion, which includes supplementary Refs. [34-61].

[34] J. Deslippe, G. Samsonidze, D. A. Strubbe, M. Jain, M. L. Cohen, and S. G. Louie, Comput. Phys. Commun. 183, 1269 (2012).

[35] E. Baldini, A. Dominguez, L. Chiodo, E. Sheveleva, M. Yazdi-Rizi, C. Bernhard, A. Rubio, and M. Chergui, Phys. Rev. B 96, 041204(R) (2017).

[36] H. Berger, H. Tang, and F. Levy, J. Cryst. Growth 130, 108 (1993).

[37] M. Dressel and G. Grüner, Electrodynamics of Solids: Optical Properties of Electrons in Matter (Cambridge University Press, Cambridge, England, 2002).

[38] E. Baldini, M. A. Sentef, S. Acharya, T. Brumme, E. Sheveleva, F. Lyzwa, E. Pomjakushina, C. Bernhard, M. van Schilfgaarde, F. Carbone, A. Rubio, and C. Weber, Proc. Natl. Acad. Sci. U.S.A. 117, 6409 (2020).

[39] F. Novelli, D. Fausti, J. Reul, F. Cilento, P. H. M. Van Loosdrecht, A. A. Nugroho, T. T. M. Palstra, M. Grüninger, and F. Parmigiani, Phys. Rev. B 86, 165135 (2012).

[40] H. Matsuzaki, Y. Matsui, R. Uchida, H. Yada, T. Terashige, B.-S. Li, A. Sawa, M. Kawasaki, Y. Tokura, and H. Okamoto, J. Appl. Phys. 115, 053514 (2014).

[41] M. A. M. Versteegh, T. Kuis, H. T. C. Stoof, and J. I. Dijkhuis, Phys. Rev. B 84, 035207 (2011).

[42] M. Nagai, R. Shimano, K. Horiuch, and M. KuwataGonokami, Phys. Status Solidi B 238, 509 (2003).

[43] G. Manzke, D. Semkat, F. Richter, D. Kremp, and K. Henneberger, J. Phys. Conf. Ser. 210, 012020 (2010).

[44] J. Heckötter, M. Freitag, D. Fröhlich, M. Aßmann, M. Bayer, P. Grünwald, F. Schöne, D. Semkat, H. Stolz, and S. Scheel, Phys. Rev. Lett. 121, 097401 (2018).
[45] P. P. Altermatt, J. Schmidt, G. Heiser, and A. G. Aberle, J. Appl. Phys. 82, 4938 (1997).

[46] R. Hull, Properties of Crystalline Silicon (IET, London, 1999), Vol. 20.

[47] M. A. Green, AIP Adv. 3, 112104 (2013).

[48] F. Majumder, H. Kalt, C. Klingshirn, H. Stanzl, and W. Gebhardt, Phys. Status Solidi B 188, 191 (1995).

[49] Y.-M. Yu, S. Nam, K.-S. Lee, Y. D. Choi, and B. O, J. Appl. Phys. 90, 807 (2001).

[50] A. Manar, A. Chergui, D. Guennani, D. Ohlmann, T. Cloitre, and R. L. Aulombard, Mater. Sci. Eng. B 43, 121 (1997).

[51] D. Semkat, F. Richter, D. Kremp, G. Manzke, W.-D. Kraeft, and K. Henneberger, Phys. Rev. B 80, 155201 (2009).

[52] F. Henneberger, U. Woggon, J. Puls, and C. Spiegelberg, Appl. Phys. B 46, 19 (1988).

[53] C. Fricke, R. Heitz, A. Hoffmann, and I. Broser, Phys. Rev. B 49, 5313 (1994).

[54] J. G. Fujimoto, S. G. Shevel, and E. P. Ippen, Solid State Commun. 49, 605 (1984).

[55] O. Maksimov, M. Muñoz, and M. Tamargo, Microelectron. J. 37, 19 (2006).

[56] S. Z. Karazhanov, Y. Zhang, A. Mascarenhas, and S. Deb, J. Appl. Phys. 87, 8786 (2000).

[57] H. Deng, G. Weihs, C. Santori, J. Bloch, and Y. Yamamoto, Science 298, 199 (2002).

[58] J. D. Plumhof, T. Stöferle, L. Mai, U. Scherf, and R. F. Mahrt, Nat. Mater. 13, 247 (2014).

[59] K. S. Daskalakis, S. A. Maier, R. Murray, and S. Kéna-Cohen, Nat. Mater. 13, 271 (2014).

[60] D. Varsano, G. Giorgi, K. Yamashita, and M. Palummo, J. Phys. Chem. Lett. 8, 3867 (2017).

[61] T. Hitosugi, Y. Furubayashi, A. Ueda, K. Itabashi, K. Inaba, Y. Hirose, G. Kinoda, Y. Yamamoto, T. Shimada, and T. Hasegawa, Jpn. J. Appl. Phys. 44, L1063 (2005).

[62] A. Amo, M. D. Martín, L. Viña, A. I. Toropov, and K. S. Zhuravlev, Phys. Rev. B 73, 035205 (2006).

[63] C. Klingshirn, R. Hauschild, J. Fallert, and H. Kalt, Phys. Rev. B 75, 115203 (2007).

[64] H. Watanabe and H. Okushi, Jpn. J. Appl. Phys. 39, L835 (2000).

[65] C. Choi, Y. H. Kwon, J. S. Krasinski, G. H. Park, G. Setlur, J. J. Song, and Y. C. Chang, Phys. Rev. B 63, 115315 (2001).

[66] H. Haug and S. Schmitt-Rink, J. Opt. Soc. Am. B 2, 1135 (1985).

[67] S. Schmitt-Rink, D. S. Chemla, and D. A. B. Miller, Adv. Phys. 38, 89 (1989).

[68] E. Burstein, Phys. Rev. 93, 632 (1954).

[69] T. S. Moss, Proc. Phys. Soc. London Sect. B 67, 775 (1954).

[70] E. M. Bothschafter, A. Paarmann, E. S. Zijlstra, N. Karpowicz, M. E. Garcia, R. Kienberger, and R. Ernstorfer, Phys. Rev. Lett. 110, 067402 (2013).

[71] E. Baldini, T. Palmieri, E. Pomarico, G. Auböck, and M. Chergui, ACS Photonics 5, 1241 (2018).

[72] P. K. Gogoi, L. Sponza, D. Schmidt, T. C. Asmara, C. Diao, J. C. W. Lim, S. M. Poh, S.-i. Kimura, P. E. Trevisanutto, V. Olevano, and A. Rusydi, Phys. Rev. B 92, 035119 (2015).

[73] V. Begum, M. E. Gruner, and R. Pentcheva, Phys. Rev. Mater. 3, 065004 (2019).

[74] C. Rödl and F. Bechstedt, Phys. Rev. B 86, 235122 (2012). 